\title{
Isolation of Neutrophil Nuclei for Use in NETosis Assays
}

Dave Boucher*

Institut de Biochimie, Université de Lausanne, Epalinges, Switzerland

*For correspondence: dave.boucher@unil.ch

[Abstract] Neutrophils are critical immune cells that protect our body against invading pathogens. They generate antibacterial DNA structures called neutrophil extracellular traps (NET). Recently we identified a new mechanism that enables NET formation. We observed that following recognition of lipopolysaccharides, inflammatory caspases cleave Gasdermin D and enable NET generation (Chen et al., 2018). This protocol describes how we purify neutrophil nuclei to visualize NET formation by live microscopy. After neutrophil purification from murine bone marrow, neutrophils are lysed in a hypotonic buffer using a nitrogen cavitation device to prevent lysis of neutrophil granules and subsequent contamination by granules proteases. Lysed neutrophils are then centrifuged, and nuclei are counted. The protocol described here is straightforward and enables the study of early changes happening in the nuclei of neutrophils undergoing NETosis with limited contamination by granule proteases.

Keywords: Neutrophil extracellular trap (NET), Cell death, Host-Pathogens interactions, Neutrophils, Caspases

[Background] NETosis is a neutrophil-specific host-defense mechanism that enables DNA extrusion to trap bacteria during infection (Papayannopoulos, 2018). This process is associated with dramatic changes in neutrophil nucleus structure and the release of DNA and chromatin outside the neutrophil. NETosis is studied mostly by microscopy using live or fixed neutrophils stimulated with various triggers like bacterium or Phorbol 12-myristate 13-acetate (Gonzalez et al., 2014). We recently described that netosis can be activated upon detection of intracellular LPS by the noncanonical inflammasome (Chen et al., 2018). Using purified neutrophil nuclei, we showed that incubation of nuclei with recombinant Gasdermin D (Shi et al., 2015), a pore-forming protein, and the inflammatory caspase-11, is sufficient to trigger nuclear changes that are associated with NETosis. Here we offer a simple framework to study NET generation on purified nuclei to visualize early changes happening before and during DNA extrusion. Our protocol enables the study of NET formation without contamination by neutrophil proteases [which can also trigger NET formation (Papayannopoulos, 2018)] and was developed to study the role of Gasdermin D during the early step of NET formation.

\section{Materials and Reagents}

1. Pipette tips $(1000 \mu \mathrm{l}, 200 \mu \mathrm{l}, 10 \mu \mathrm{l})$

2. Tissue, Kimwipes disposable wipers (Sigma-Aldrich, catalog number: Z188956-1PAK)

3. 6-well plate, sterile tissue culture grade (Sigma-Aldrich, Corning, catalog number: SIAL0506- 
100EA)

4. $15 \mathrm{ml}$ sterile conical tubes (Sigma-Aldrich, Greiner, catalog number: T1943-1000EA)

5. $1.5 \mathrm{ml}$ tubes sterile (Eppendorf, catalog number: 0030 120.086)

6. $27 \mathrm{G}$ needle (Sigma-Aldrich, catalog number: Z192384-100EA)

7. $10 \mathrm{ml}$ disposable plastic syringe (Fischer Scientific, catalog number: 15889152)

8. 12 weeks-old mouse C57BL/6J

9. EasySep neutrophil enrichment kit (StemCell Technology, catalog number: 19762)

10. Ethanol

11. RPMI 1640 (Thermo Fisher Scientific, Gibco, catalog number: 61870010)

12. TC grade $1 \mathrm{M}$ HEPES (Thermo Fisher Scientific, Gibco, catalog number: 15630080)

13. Trypan blue solution (Thermo Fisher Scientific, catalog number: 15250061)

14. ATP sodium (Sigma-Aldrich, catalog number: A7699-1G)

15. Magnesium Chloride $\left(\mathrm{MgCl}_{2}\right)$ (Sigma-Aldrich, catalog number: M2670)

16. Potassium Chloride (KCl) (Sigma-Aldrich, catalog number: P9333)

17. Phenylmethanesulfonyl fluoride (PMSF) (Sigma-Aldrich, Roche, catalog number: PMSF-RO)

18. cOmplete Mini, EDTA-free protease inhibitor tablets (Sigma-Aldrich, Roche, catalog number: 11836170001)

19. Opti-MEM, reduced-serum media (Thermo Fisher Scientific, catalog number: 31985062 )

20. $70 \%$ Ethanol solution (see Recipes)

21. Neutrophil media (see Recipes)

22. PMSF stock solution (see Recipes)

23. ATP stock solution (see Recipes)

24. Hypotonic buffer (see Recipes)

\section{Equipment}

1. P1000 micropipette

2. Scissors

3. Hematocytometer

4. Centrifuge (Eppendorf, model: $5415 \mathrm{C}$ )

5. Incubator

6. $-80^{\circ} \mathrm{C}$ freezer

7. Cell disruption vessel, $45 \mathrm{ml}$ (Parr Instrument Company, catalog number: 4639)

8. Nitrogen tank linked to a pressure-reducing valve

9. Mouse Dissecting kit (World Precision Instrument)

10. Tissue culture hood 


\section{Procedure}

1. Kill the mice by cervical dislocation or any other suitable method.

Note: Protocols used to euthanize the mouse should be approved by your institution animal ethics committee.

2. Pin the mouse on its back using needles. Pin each leg. Spray the mouse with $70 \%$ ethanol. Using the mouse dissection kit, cut the skin along the anterior legs to access the tibia and the femur.

3. Cut the anterior legs just above the hips and the ankles.

4. Using a tissue, gently remove the flesh around the bones. Separate the femur and the tibia. Place the bones for $15 \mathrm{~s}$ in $70 \%$ ethanol solution in a 6 -well plate. Bring the bones (while in ethanol) inside the cell culture hood. Transfer the bones into neutrophil media in a 6-well plate and keep them on ice.

5. Under the hood, cut the extremity of the bones with a pair of scissors and flush the bone with neutrophil media using a $27 \mathrm{G}$ needle.

Note: Usually, $10 \mathrm{ml}$ of neutrophil media per mouse is sufficient for flushing.

6. Purify bone-marrow neutrophils using a negative-selection kit EasySep from StemCell Technology. Alternatively, neutrophils can also be purified as described in (Chen et al., 2014). Keep the cells on ice upon elution.

Note: Routinely, a 12 weeks-old mouse should give you between 12 and 20 millions of neutrophils. Male mice will yield a higher neutrophil count.

7. Count the viable cells using a hematocytometer by diluting the eluate 1 in 10 with trypan blue.

8. Centrifuge the cells at $400 \times \mathrm{g}$ for $5 \mathrm{~min}$ at $4{ }^{\circ} \mathrm{C}$. Remove the cell supernatant by aspiration.

9. Using a P1000 micropipette, gently resuspend the neutrophil to a concentration of 50 million neutrophils/ml of hypotonic buffer.

Note: Neutrophils are very fragile cells. Treat them gently and avoid vortexing them or resuspending by shaking them. Such treatment can activate neutrophils and will affect the outcome of your experiment. We usually resuspend cells slowly using a P1000 micropipette.

10. Incubate the cells on ice for $10 \mathrm{~min}$ to let them swell.

11. While the cells are lysing, prepare the nitrogen cavitation device (Zhou and Philipp, 2017). Make sure that the lower valve of the vessel is closed.

12. Remove the upper part of the cell disruption vessel and add your cells inside. Keep the vessel on ice.

13. Connect the vessel to the nitrogen tank and gently open the pressure-reducing valve on the tank until the indicator on the vessel indicates $380 \mathrm{psi}$. Close the upper valve and disconnect the nitrogen tank. Incubate on ice for $5 \mathrm{~min}$.

Note: Carefully monitor the pressure. Too high pressure may disrupt neutrophil granules and contaminate your nuclei preparation.

14. Take a $1.5 \mathrm{ml}$ microfuge tube and place it close to the lower valve. Slowly open the valves and 
let the liquid flow inside the microtube.

Note: Be careful when opening the lower valve. Opening the valve too quickly can result in a complete sample loss.

15. Centrifuge the cell extract for $5 \mathrm{~min}$ at $500 \times \mathrm{g}, 4^{\circ} \mathrm{C}$. Gently remove the supernatant.

16. Add $300 \mu$ l of hypotonic buffer to the pellet and gently resuspend it. Repeat Step 15 twice.

17. Resuspend gently your pellet in $200 \mu \mathrm{l}$ of the hypotonic buffer using a P1000 pipette. Using a hematocytometer, count the nuclei and resuspend them according to your need.

18. The nuclei preparation is ready to be used for your assay.

Notes:

a. The nuclei preparation should be prepared for immediate use. It is not recommended to freeze if the preparation is meant to be used for NETosis studies.

b. This protocol can be used to purify nuclei from neutrophils isolated from fresh human blood. In that case, experimenter should start the protocol from Step 7. The EasySep human neutrophil enrichment kit (StemCell Technology) can be used to purify human neutrophils.

c. Final resuspension (step 17) can be performed in other buffers if those are more convenient for the experimenter. Such buffer may not lyse the nucleus and must be tested before used to ensure that the nucleus stays intact (e.g., not permeable to SYTOX staining). See the Data analysis section for further details.

\section{Data analysis}

This protocol should enable the purification of intact neutrophil nuclei. If used for microscopy, the experimenter should verify that the nuclei are intact and not generating NET using a DNA staining reagent (e.g., SYTOX, Invitrogen) (Chen et al., 2018).

\section{$\underline{\text { Recipes }}$}

1. $70 \%$ Ethanol solution

Prepare $70 \%$ ethanol solution with distilled water

2. Neutrophil media

$500 \mathrm{ml}$ RPMI 1640

$10 \%$ Fetal Calf Serum (Heat-inactivated)

$5 \mathrm{ml}$ TC grade $1 \mathrm{M}$ HEPES

Keep the reconstituted media at $4{ }^{\circ} \mathrm{C}$, away from light

3. PMSF stock solution
a. Dissolve in ethanol
b. Aliquot the solution into $1 \mathrm{ml}$ tubes
C. Store at $-20^{\circ} \mathrm{C}$

4. ATP stock solution $(0.5 \mathrm{M})$ 
a. Dissolve ATP in sterile distilled water

b. Aliquot on ice and freeze at $-80^{\circ} \mathrm{C}$

Note: Do not freeze and thaw the aliquot, discard after thawing.

5. Hypotonic buffer

$100 \mathrm{mM} \mathrm{KCl}$

$1 \mathrm{mM} \mathrm{NaCl}$

$1 \mathrm{mM}$ ATP

$3.5 \mathrm{mM} \mathrm{MgCl} 2$

$10 \mathrm{mM}$ PIPES

1 Tabs of cOmpete Mini EDTA-free protease inhibitor ( $1 \mathrm{tab} / 5 \mathrm{ml}$ of buffer)

$0.5 \mathrm{mM}$ PMSF

Note: The ATP, the protease inhibitor tablets, and the PMSF should be added freshly (10 min before lysing the cells).

\section{Acknowledgments}

During the optimization of this protocol, Dr. Dave Boucher was supported by a postdoctoral fellowship from the University of Queensland. The author thanks Pr. Kate Schroder and Dr. Ian Ross (University of Queensland) for their assistance while optimizing this protocol.

\section{Competing interests}

The author has no competing interests to declare.

\section{Ethics}

The University of Queensland's animal ethics committee approved all experimental protocols (approval number IMB/423/17).

\section{References}

1. Chen, K. W., Gross, C. J., Sotomayor, F. V., Stacey, K. J., Tschopp, J., Sweet, M. J. and Schroder, K. (2014). The neutrophil NLRC4 inflammasome selectively promotes IL-1beta maturation without pyroptosis during acute Salmonella challenge. Cell Rep 8(2): 570-582.

2. Chen, K. W., Monteleone, M., Boucher, D., Sollberger, G., Ramnath, D., Condon, N. D., von Pein, J. B., Broz, P., Sweet, M. J. and Schroder, K. (2018). Noncanonical inflammasome signaling elicits gasdermin D-dependent neutrophil extracellular traps. Sci Immunol 3(26). pii: eaar6676. 
3. Gonzalez, A. S., Bardoel, B. W., Harbort, C. J. and Zychlinsky, A. (2014). Induction and quantification of neutrophil extracellular traps. Methods Mol Biol 1124: 307-318.

4. Papayannopoulos, V. (2018). Neutrophil extracellular traps in immunity and disease. Nat Rev Immunol 18(2): 134-147.

5. Shi, J., Zhao, Y., Wang, K., Shi, X., Wang, Y., Huang, H., Zhuang, Y., Cai, T., Wang, F. and Shao, F. (2015). Cleavage of GSDMD by inflammatory caspases determines pyroptotic cell death. Nature 526(7575): 660-665.

6. Zhou, M. and Philips, M. R. (2017). Nitrogen cavitation and differential centrifugation allows for monitoring the distribution of peripheral membrane proteins in cultured cells. $J$ Vis Exp 18(126). doi: $10.3791 / 56037$. 